\title{
Three-form multiplet and supersymmetry breaking
}

\section{Evgeny I. Buchbinder and Sergei M. Kuzenko}

School of Physics and Astrophysics M013, The University of Western Australia, 35 Stirling Highway, Crawley W.A. 6009, Australia

E-mail: evgeny.buchbinder@uwa.edu.au, sergei.kuzenko@uwa.edu.au

ABSTRACT: Recently, a nilpotent real scalar superfield $V$ was introduced in [1] as a model for the Goldstino. It contains only two independent component fields, the Goldstino and the auxiliary $D$-field. Here we first show that $V$ can equivalently be realised as a constrained three-form superfield. We demonstrate that every irreducible Goldstino superfield (of which the Goldstino is the only independent component field) can be realised as a descendant of $V$ which is invariant under local rescalings $V \rightarrow \mathrm{e}^{\tau} V$, where $\tau$ is an arbitrary real scalar superfield. We next propose a new Goldstino supermultiplet which is described by a nilpotent three-form superfield $\mathcal{Y}$ that is a variant formulation for the nilpotent chiral superfield, which is often used in off-shell models for spontaneously broken supergravity. It is shown that the action describing the dynamics of $\mathcal{Y}$ may be obtained from a supersymmetric nonlinear $\sigma$-model in the infrared limit. Unlike $V$, the Goldstino superfield $\mathcal{Y}$ contains two independent auxiliary fields, $F=H+\mathrm{i} G$, of which $H$ is a scalar and $G$ is the field strength of a gauge three-form. When $\mathcal{Y}$ is coupled to supergravity, both $H$ and $G$ produce positive contributions to the cosmological constant. While the contribution from $H$ is uniquely determined by the parameter of the supersymmetry breaking in the action, the contribution from $G$ is dynamical.

KEYWORDS: Superspaces, Supersymmetry Breaking

ARXIV EPRINT: 1705.07700 


\section{Contents}

1 Introduction 1

2 From $V$ to equivalent three-form multiplet 3

3 Irreducible and reducible Goldstino superfields 5

4 Nilpotent three-form Goldstino multiplet $\quad 6$

5 Models for spontaneously broken supergravity $\quad 9$

$\begin{array}{llr}5.1 & \text { Super-Weyl inert } V & 10\end{array}$

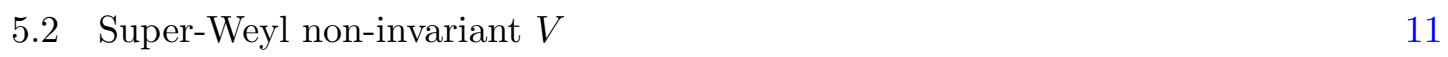

$\begin{array}{lll}5.3 & \text { Three-form supergravity } & 11\end{array}$

$\begin{array}{lll}5.4 & \text { Three-form Goldstino superfield in supergravity } & 12\end{array}$

\section{Introduction}

Recently, a new Goldstino superfield for spontaneously broken $\mathcal{N}=1$ supersymmetry in four spacetime dimensions has been introduced [1]. In the super-Poincaré case, it is described by a real scalar superfield $V$ subject to the nilpotency constraints

$$
\begin{aligned}
V^{2} & =0, \\
V D_{A} D_{B} V & =0, \\
V D_{A} D_{B} D_{C} V & =0,
\end{aligned}
$$

where $D_{A}=\left(\partial_{a}, D_{\alpha}, \bar{D}^{\dot{\alpha}}\right)$ are the covariant derivatives of $\mathcal{N}=1$ Minkowski superspace $\mathbb{M}^{4 \mid 4}$. These nilpotency constraints have to be supplemented with the requirement that the descendant $D^{\alpha} W_{\alpha}=\bar{D}_{\dot{\alpha}} \bar{W}^{\dot{\alpha}}$ is nowhere vanishing, where

$$
W_{\alpha}=-\frac{1}{4} \bar{D}^{2} D_{\alpha} V
$$

The nilpotency constraints (1.1) imply that $V$ has two independent component fields ${ }^{1}$ which are: the Goldstino, which may be identified with $\left.W_{\alpha}\right|_{\theta=0}$, and the auxiliary $D$-field being proportional to $\left.D^{\alpha} W_{\alpha}\right|_{\theta=0}$. All other component fields of $V$ are composite ones. In particular, from (1.1) one may deduce the representation

$$
V=-4 \frac{W^{2} \bar{W}^{2}}{\left(D^{\alpha} W_{\alpha}\right)^{3}}, \quad W^{2}=W^{\alpha} W_{\alpha}
$$

\footnotetext{
${ }^{1}$ The component analysis of $V$ simplifies if one notices that the constraints (1.1b) and (1.1c) are equivalent to $V \partial_{A} \partial_{B} V=0$ and $V \partial_{A} \partial_{B} \partial_{C} V=0$, respectively.
} 
which turns (1.1) into identities. The dynamics of this supermultiplet is governed by the action

$$
S=\int \mathrm{d}^{4} x \mathrm{~d}^{2} \theta \mathrm{d}^{2} \bar{\theta}\left\{\frac{1}{16} V D^{\alpha} \bar{D}^{2} D_{\alpha} V-2 f V\right\}
$$

where $f$ is a positive parameter of mass dimension +2 which characterises the supersymmetry breaking scale.

The constraints (1.1) are invariant under local re-scalings of $V$,

$$
V \rightarrow \mathrm{e}^{\tau} V
$$

where $\tau$ is an arbitrary real scalar superfield. Requiring the action (1.4) to be stationary under arbitrary variations of the form $\delta V=\tau V$ leads to the following constraint

$$
f V=\frac{1}{16} V D^{\alpha} \bar{D}^{2} D_{\alpha} V
$$

which proves to express the auxiliary field in terms of the Goldstino. The constraints (1.1) and (1.6) define the irreducible Goldstino superfield $\mathcal{V}$ described in [2].

An explicit realisation for $\mathcal{V}$ was given long ago by Lindström and Roček [3] in the form

$$
f \mathcal{V}=\bar{\phi} \phi
$$

where $\phi$ is the irreducible chiral scalar Goldstino superfield $[4,5], \bar{D}_{\dot{\alpha}} \phi=0$, which is subject to the constraints [4]:

$$
\begin{aligned}
\phi^{2} & =0 \\
f \phi & =-\frac{1}{4} \phi \bar{D}^{2} \bar{\phi} .
\end{aligned}
$$

Another useful realisation for $\mathcal{V}$ is

$$
f \mathcal{V}=\bar{\Sigma} \Sigma,
$$

where $\Sigma$ is the Goldstino superfield introduced in [6]. It obeys the improved complex linear constraint

$$
-\frac{1}{4} \bar{D}^{2} \Sigma=f
$$

as well as it is nilpotent and is subject to a holomorphic nonlinear constraint,

$$
\begin{aligned}
\Sigma^{2} & =0, \\
f D_{\alpha} \Sigma & =-\frac{1}{4} \Sigma \bar{D}^{2} D_{\alpha} \Sigma .
\end{aligned}
$$

In this paper we uncover new interesting properties of the Goldstino multiplet $V$. In particular, we show that $V$ can equivalently be realised as a constrained three-form superfield. We also demonstrate that every irreducible Goldstino superfield ${ }^{2}$ can be realised

\footnotetext{
${ }^{2}$ The notion of irreducible and reducible Goldstino superfields was introduced in [2], see also section 3 below.
} 
as a descendant of $V$, which is invariant under local rescalings $V \rightarrow \mathrm{e}^{\tau} V$, where $\tau$ is an arbitrary real scalar superfield.

In the last two years, there has been much interest in models for off-shell $\mathcal{N}=1$ supergravity coupled to nilpotent Goldstino superfields. One of the main reasons for such interest is that every Goldstino superfield coupled to supergravity provides a positive contribution to the cosmological constant [2,3,8-11], unlike the supersymmetric cosmological term [12] which yields a negative contribution to the cosmological constant. The same property holds for the Goldstino brane [13]. All irreducible Goldstino superfields provide one and the same universal contribution to the cosmological constant [2], in accordance with the super-Higgs effect [14-16]. Exactly the same contribution is generated by the known reducible Goldstino multiplets, which are: (i) the superfield $V$ discussed above; and (ii) the nilpotent chiral superfield $\mathcal{X}[17,18]$ used in numerous recent publications including [8-10]. In this paper we introduce a new reducible Goldstino superfield, a nilpotent three-form multiplet, which will be shown to provide two separate positive contributions to the cosmological constant, of which one is universal and the other is dynamical.

\section{From $V$ to equivalent three-form multiplet}

We start by recalling two simple models for spontaneously broken supersymmetry that are realised in terms of an unconstrained real scalar superfield $V$. One of them describes an abelian vector multiplet with action

$$
S_{\mathrm{VM}}=\left\{\frac{1}{8} \int \mathrm{d}^{4} x \mathrm{~d}^{2} \theta W^{\alpha} W_{\alpha}+\text { c.c. }\right\}-2 f \int \mathrm{d}^{4} x \mathrm{~d}^{2} \theta \mathrm{d}^{2} \bar{\theta} V,
$$

which is obtained by adding a Fayet-Iliopoulos term [7] to the massless vector multiplet action. The functional (2.1) is invariant under gauge transformations

$$
\delta V=\lambda+\bar{\lambda}, \quad \bar{D}_{\dot{\alpha}} \lambda=0 .
$$

The chiral spinor $W_{\alpha}$ defined by (1.2) is a gauge-invariant field strength for this model.

Our second model describes the dynamics of a variant scalar multiplet known as the three-form multiplet $[19,20]$ (see [21] for a review). We choose the action

$$
S_{\mathrm{TFM}}=\int \mathrm{d}^{4} x \mathrm{~d}^{2} \theta \mathrm{d}^{2} \bar{\theta} \bar{\Psi} \Psi-\left\{f \int \mathrm{d}^{4} x \mathrm{~d}^{2} \theta \Psi+\text { c.c. }\right\},
$$

where the chiral scalar superfield $\Psi, \bar{D}_{\dot{\alpha}} \Psi=0$, is defined by

$$
\Psi=-\frac{1}{4} \bar{D}^{2} V
$$

It is called the three-form multiplet. Its specific feature is the relation

$$
D^{2} \Psi-\bar{D}^{2} \bar{\Psi}=\mathrm{i} \partial^{\alpha \dot{\alpha}} v_{\alpha \dot{\alpha}}, \quad v_{\alpha \dot{\alpha}}=\left[D_{\alpha}, \bar{D}_{\dot{\alpha}}\right] V,
$$

which means that the auxiliary $F$-field of $\Psi$, defined by $F=-\left.\frac{1}{4} D^{2} \Psi\right|_{\theta=0}$, is a complex scalar such that its imaginary part, $\operatorname{Im} F$, is the divergence of a vector (or, equivalently, the 
field strength of a gauge three-form). This is the only difference between the three-form multiplet and the standard scalar multipelt.

The field strength (2.4) and the action (2.3) are invariant under gauge transformations

$$
\delta V=L, \quad \bar{L}=L, \quad \bar{D}^{2} L=0,
$$

where $L$ is an arbitrary linear multiplet. This gauge symmetry corresponds to a gauge theory with linearly dependent generators, following the terminology of the Batalin-Vilkovisky quantisation [22], and therefore the quantisation of (2.3) cannot be carried out using the Faddeev-Popov procedure. ${ }^{3}$

The supersymmetric theories (2.1) and (2.3) describe the dynamics of two different multiplets, the vector and the scalar ones, respectively, if the dynamical superfield $V$ is unconstrained. However, in case $V$ is subject to the nilpotency constraints (1.1), which are incompatible with the gauge symmetries (2.2) and (2.6), any difference between the actions (2.1) and (2.3) proves to disappear. Once the constraints (1.1) are taken into account, the action (2.1) (which coincides with the right-hand side of (1.4) modulo a total derivative) can be rewritten in the form (2.3). As a consequence of (1.1), the chiral scalar $\Psi$ proves to be nilpotent,

$$
\Psi^{2}=0 \quad \Longrightarrow \quad \Psi=-\frac{D^{\alpha} \Psi D_{\alpha} \Psi}{D^{2} \Psi} .
$$

It also obeys the nonlinear constraint

$$
\Psi=2 \bar{D}^{2} \frac{\bar{\Psi} \Psi}{D^{2} \Psi+\bar{D}^{2} \bar{\Psi}}
$$

which expresses the field strength of the gauge three-form, $\operatorname{Im} F$, in terms of the Goldstino and $\operatorname{Re} F$. The latter constraint follows from the observation that (1.3) may be rewritten in several equivalent forms

$$
V=-4 \frac{\bar{\Psi} \Psi}{\bar{D}^{2} \bar{\Psi}}=-4 \frac{\bar{\Psi} \Psi}{D^{2} \Psi}=-8 \frac{\bar{\Psi} \Psi}{D^{2} \Psi+\bar{D}^{2} \bar{\Psi}}
$$

The above analysis demonstrates that the Goldstino multiplet $V$ under the nilpotency conditions (1.1) is equivalent to the chiral superfield $\Psi$ subject to the constraints (2.7). Therefore, the description in terms of $\Psi$ provides a different realisation of the same multiplet.

It is also worth pointing out that the chiral superfield $\phi$ defined by (1.8) is the threeform multiplet associated with the irreducible Goldstino superfield $\mathcal{V}$ defined by the constraints (1.1) and (1.6),

$$
\phi=-\frac{1}{4} \bar{D}^{2} \mathcal{V}
$$

\footnotetext{
${ }^{3}$ Covariant quantisation of models for the three-form multiplet coupled to supergravity was carried out in [23] (see [24] for a review).
} 


\section{$3 \quad$ Irreducible and reducible Goldstino superfields}

In accordance with the discussion in [2], there are two general types of $\mathcal{N}=1$ Goldstino superfields, irreducible and reducible ones. Every irreducible Goldstino superfield contains only one independent component field - the Goldstino itself, while the other component fields are composites constructed from the Goldstino. ${ }^{4}$ Given an irreducible Goldstino superfield, the corresponding component action can be related to the Volkov-Akulov action [25-27] using the formalism developed in [28]. Every reducible Goldstino superfield contains at least two independent fields, one of which is the Goldstino and the other fields are auxiliary (the latter become descendants of the Goldstino on the mass shell).

Examples of irreducible Goldstino superfields are the chiral scalar (1.8) and the complex linear scalar (1.10). The well-known example of a reducible Goldstino superfield was introduced in $[17,18]$. It is a chiral scalar $\mathcal{X}, \bar{D}_{\dot{\alpha}} \mathcal{X}=0$, which is subject to the nilpotency constraint

$$
\mathcal{X}^{2}=0
$$

in conjunction with the requirement that the descendant $D^{2} \mathcal{X}$ is nowhere vanishing. The dynamics of this supermultiplet is described by the action

$$
S=\int \mathrm{d}^{4} x \mathrm{~d}^{2} \theta \mathrm{d}^{2} \bar{\theta} \overline{\mathcal{X}} \mathcal{X}-\left\{f \int \mathrm{d}^{4} x \mathrm{~d}^{2} \theta \mathcal{X}+\text { c.c. }\right\} .
$$

As was pointed out in [2], the nilpotency condition (3.1) is preserved if $\mathcal{X}$ is locally rescaled,

$$
\mathcal{X} \rightarrow \mathrm{e}^{\tau} \mathcal{X}, \quad \bar{D}_{\dot{\alpha}} \tau=0
$$

Requiring the action (3.2) to be stationary under such re-scalings of $\mathcal{X}$ (see [1]) gives

$$
\mathcal{X}=\phi
$$

where $\phi$ is Roček's chiral scalar defined by (1.8).

As was demonstrated in [6] (see also [2, 29]), every irreducible Goldstino superfield can be expressed in terms of the complex linear Goldstino superfield $\Sigma$, eq. (1.10), and its conjugate $\bar{\Sigma}$. On the other hand, $\Sigma$ can be represented as a descendant of the reducible Goldstino superfield $V$ subject to the constraints (1.1). The corresponding realisation is

$$
\Sigma=-4 f \frac{D^{2} V}{\bar{D}^{2} D^{2} V}=-4 f \frac{\bar{\Psi}}{\bar{D}^{2} \bar{\Psi}} .
$$

A remarkable feature of this representation is that $\Sigma$ proves to be invariant under local re-scalings (1.5) of $V$,

$$
\delta_{\tau} V=\tau V \quad \Longrightarrow \quad \delta_{\tau} \Sigma=0 .
$$

\footnotetext{
${ }^{4}$ All known irreducible scalar Goldstino superfields are nilpotent, and the important examples are provided by eqs. (1.8a) and (1.10b).
} 
Here $\tau$ is an arbitrary real scalar superfield. Since every irreducible Goldstino superfield is a descendant of $\Sigma$ and $\bar{\Sigma}$, we conclude that all irreducible Goldstino superfields are invariant under local re-scalings (1.5) of $V$. This includes the real Goldstino superfield $\mathcal{V}$ given by (1.9), with $\Sigma$ realised as in (3.5).

Now let us note that a transformation (1.5), where $V$ is subject to (1.1) rescales the auxiliary field. Hence, the invariance of $\Sigma$ under this transformation means that $\Sigma$ does not contain the auxiliary field and describes an irreducible Goldstino model. This observation also explains why all irreducible Goldstino models (which contain only the Goldstino and no auxiliary fields) are invariant under re-scaling of $V$.

As was noticed in [2], every reducible Goldstino superfield can always be represented as an irreducible one plus a "matter" superfield, which contains all the auxiliary component fields. In our case, the reducible Goldstino superfield $V$ can be realised as

$$
V=\mathcal{V}+U, \quad \mathcal{V}=\frac{1}{f} \bar{\Sigma} \Sigma,
$$

where $\Sigma$ is given by (3.5). The "matter" superfield $U$ obeys the generalised nilpotency condition

$$
U^{2}+2 \mathcal{V} U=0
$$

and transforms under (3.6) as

$$
\delta_{\tau} U=\tau(\mathcal{V}+U)
$$

The superfield $U$ contains only one independent component field, which is the auxiliary field of $V$.

It should be remarked that $\Sigma$ can also be expressed in terms of $\overline{\mathcal{X}}$ as

$$
\Sigma=-4 f \frac{\overline{\mathcal{X}}}{\bar{D}^{2} \overline{\mathcal{X}}}
$$

compare with (3.5). One may check that $\Sigma$ is invariant under arbitrary re-scalings (3.3). This implies that all irreducible Goldstino superfields, realised as descendants of $\mathcal{X}$ and $\overline{\mathcal{X}}$, are invariant under local re-scalings (3.3) of $\mathcal{X}$.

\section{Nilpotent three-form Goldstino multiplet}

In this section, we introduce a new reducible Goldstino superfield. It is a three-form multiplet,

$$
\mathcal{Y}=-\frac{1}{4} \bar{D}^{2} \mathcal{U}, \quad \overline{\mathcal{U}}=\mathcal{U},
$$

which is constrained to be nilpotent,

$$
\mathcal{Y}^{2}=0
$$


in conjunction with the requirement that the descendant $D^{2} \mathcal{Y}$ is nowhere vanishing. The relations (4.1) are invariant under gauge transformations of the type (2.6). The dynamics of $\mathcal{Y}$ is described by the action

$$
S=\int \mathrm{d}^{4} x \mathrm{~d}^{2} \theta \mathrm{d}^{2} \bar{\theta} \overline{\mathcal{Y}} \mathcal{Y}-\left\{h \int \mathrm{d}^{4} x \mathrm{~d}^{2} \theta \mathcal{Y}+\text { c.c. }\right\}
$$

with $h=\bar{h}$ a positive parameter. This nilpotent three-form multiplet is a variant formulation of the nilpotent chiral multiplet $\mathcal{X}$ discussed in the previous section.

Interesting enough, the above theory can be obtained from a nonlinear $\sigma$-model action

$$
S=\int \mathrm{d}^{4} x \mathrm{~d}^{2} \theta \mathrm{d}^{2} \bar{\theta} K(\overline{\mathcal{Y}}, \mathcal{Y})+\left\{\int \mathrm{d}^{4} x \mathrm{~d}^{2} \theta W(\mathcal{Y})+\text { c.c. }\right\}
$$

in the infrared limit, as an extension of the approach advocated in [17]. Let us define the components of $\mathcal{Y}$ as

$$
\mathcal{Y}\left|=\varphi, \quad D_{\alpha} \mathcal{Y}\right|=\sqrt{2} \psi_{\alpha}, \quad-\frac{1}{4} D^{2} \mathcal{Y} \mid=F=H+\mathrm{i} G .
$$

As was already explained in eq. (2.5) it follows from (4.1a) that $G$ is a divergence of a vector which we denote by $C^{a}$, that is $G=\partial_{a} C^{a}$. Equivalently, $* G=d C$ for some three-form potential $C$. The action (4.3) in components is just the standard action for a supersymmetric nonlinear $\sigma$-model

$$
\begin{aligned}
S=\int \mathrm{d}^{4} x & {\left[K_{\varphi \bar{\varphi}}\left(-\partial_{a} \varphi \partial^{a} \bar{\varphi}+\frac{\mathrm{i}}{2} \partial_{\alpha \dot{\alpha}} \psi^{\alpha} \bar{\psi}^{\dot{\alpha}}-\frac{\mathrm{i}}{2} \psi^{\alpha} \partial_{\alpha \dot{\alpha}} \bar{\psi}^{\dot{\alpha}}+F \bar{F}\right)\right.} \\
+ & \frac{1}{2} K_{\varphi \varphi \bar{\varphi}}\left(\mathrm{i} \psi^{\alpha} \bar{\psi}^{\dot{\alpha}} \partial_{\alpha \dot{\alpha}} \varphi-\psi^{2} \bar{F}\right)-\frac{1}{2} K_{\varphi \bar{\varphi} \bar{\varphi}}\left(\mathrm{i} \psi^{\alpha} \bar{\psi}^{\dot{\alpha}} \partial_{\alpha \dot{\alpha}} \bar{\varphi}+\bar{\psi}^{2} F\right)+\frac{1}{4} K_{\varphi \varphi \bar{\varphi} \bar{\varphi}} \psi^{2} \bar{\psi}^{2} \\
& \left.-\frac{1}{2} W_{\varphi \varphi} \psi^{2}-\frac{1}{2} \bar{W}_{\bar{\varphi} \bar{\varphi}} \bar{\psi}^{2}+W_{\varphi} F+\bar{W}_{\bar{\varphi}} \bar{F}\right] .
\end{aligned}
$$

To achieve gauge invariant boundary conditions

$$
\left.\delta G\right|_{\text {boundary }}=0
$$

this action has to be supplemented with the boundary term ${ }^{5}[30,31]$

$$
S_{\text {boundary }}=-\int \mathrm{d}^{4} x \partial_{a}\left[2 K_{\varphi \bar{\varphi}} G C^{a}+\mathrm{i}\left(W_{\varphi}-\bar{W}_{\bar{\varphi}}\right) C^{a}\right] .
$$

The equations of motion for the auxiliary fields $H$ and $C^{a}$ are given by

$$
2 K_{\varphi \bar{\varphi}} H+W_{\varphi}+\bar{W}_{\bar{\varphi}}=0, \quad \partial_{a}\left(K_{\varphi \bar{\varphi}} G+\frac{\mathrm{i}}{2}\left(W_{\varphi}-\bar{W}_{\bar{\varphi}}\right)\right)=0,
$$

which lead to

$$
H=-\frac{1}{2} K_{\varphi \bar{\varphi}}^{-1}\left(W_{\varphi}+\bar{W}_{\bar{\varphi}}\right), \quad G=-\frac{\mathrm{i}}{2} K_{\varphi \bar{\varphi}}^{-1}\left(W_{\varphi}-\bar{W}_{\bar{\varphi}}\right)+K_{\varphi \bar{\varphi}}^{-1} g,
$$

\footnotetext{
${ }^{5}$ We will not discuss the supersymmetric completion of (4.7) in this note.
} 
where $g$ is an arbitrary constant. Substituting (4.9) into the action (4.5), (4.7) we obtain the scalar potential

$$
V(\varphi, \bar{\varphi})=K_{\varphi \bar{\varphi}}^{-1}\left(W_{\varphi}+\mathrm{i} g\right)\left(\bar{W}_{\bar{\varphi}}-\mathrm{i} g\right) .
$$

Note that it receives contributions from both the bulk and boundary actions. Let us choose Kähler normal coordinates in the $\sigma$-model target space near the vacuum so that in the vacuum

$$
K_{\varphi \bar{\varphi}}=1, \quad K_{\varphi \varphi \bar{\varphi}}=K_{\varphi \bar{\varphi} \bar{\varphi}}=0, \quad K_{\varphi \varphi \bar{\varphi} \bar{\varphi}}=R,
$$

where $R$ is the curvature of the target space. Then the vacuum is determined by the equation

$$
\left(\bar{W}_{\bar{\varphi}}-\mathrm{i} g\right) W_{\varphi \varphi}=0 .
$$

We are interested in a supersymmetry breaking solution for which $\langle F\rangle \sim\left\langle\bar{W}_{\bar{\varphi}}-\mathrm{i} g\right\rangle \neq 0$. This means that $\left\langle W_{\varphi \varphi}\right\rangle=0$. The vacuum energy is given by

$$
\Lambda=\langle V(\varphi, \bar{\varphi})\rangle=\left|W_{\varphi}+\mathrm{i} g\right|^{2}>0 .
$$

Since $\left\langle W_{\varphi \varphi}\right\rangle=0$, the fermion $\psi$ is massless and becomes a Goldstino. In addition, we have two massive scalar fields. For simplicity let us assume that the supersymmetry breaking vacuum is located at $\varphi=\bar{\varphi}=0$. Denoting

$$
W_{\varphi}(0)=-h=-\bar{h}, \quad \frac{1}{2} W_{\varphi \varphi \varphi}(0)=w=\bar{w},
$$

where for simplicity we have assumed that $h$ and $w$ are real, and expanding the action to quadratic order in fluctuations we find that the masses of the scalar fields are

$$
m_{ \pm}^{2}=-|\boldsymbol{f}|(|\boldsymbol{f}| R \pm w)
$$

where $\boldsymbol{f}=h+\mathrm{i} g$. To make sure that they both are positive we have to require that $R<0$.

To find the theory of just the Goldstino $\psi$ we decouple the scalar fields by taking their masses to infinity. For this we take the limit of infinite curvature $|R| \rightarrow \infty$. The leading contribution to the action (4.5) in this limit is

$$
\begin{aligned}
S_{\text {div }}=R \int \mathrm{d}^{4} x & {\left[\varphi \bar{\varphi}\left(-\partial_{a} \varphi \partial^{a} \bar{\varphi}+\frac{\mathrm{i}}{2} \partial_{\alpha \dot{\alpha}} \psi^{\alpha} \bar{\psi}^{\dot{\alpha}}-\frac{\mathrm{i}}{2} \psi^{\alpha} \partial_{\alpha \dot{\alpha}} \bar{\psi}^{\dot{\alpha}}+F \bar{F}\right)\right.} \\
+ & \left.\frac{1}{2} \bar{\varphi}\left(\mathrm{i} \psi^{\alpha} \bar{\psi}^{\dot{\alpha}} \partial_{\alpha \dot{\alpha}} \varphi-\psi^{2} \bar{F}\right)-\frac{1}{2} \varphi\left(\mathrm{i} \psi^{\alpha} \bar{\psi}^{\dot{\alpha}} \partial_{\alpha \dot{\alpha}} \bar{\varphi}+\bar{\psi}^{2} \bar{F}\right)+\frac{1}{4} \psi^{2} \bar{\psi}^{2}\right] .
\end{aligned}
$$

This gives the following equation of motion for $\phi$

$$
\frac{1}{2} \bar{\varphi} \partial_{a} \partial^{a} \varphi^{2}+\bar{F}\left(\varphi F-\frac{1}{2} \psi^{2}\right)+\mathrm{i} \partial_{\alpha \dot{\alpha}}\left(\varphi \psi^{\alpha} \bar{\psi}^{\dot{\alpha}}\right)-\mathrm{i} \varphi \psi^{\alpha} \partial_{\alpha \dot{\alpha}}\left(\bar{\psi}^{\dot{\alpha}}\right)=0
$$

It is clear that this equation is solved by

$$
\varphi=\frac{1}{2 F} \psi^{2}
$$


Note that it implies that $\varphi^{2}=\varphi \psi=0$. Eq. (4.18) is precisely what follows from the nilpotency condition (4.1b). Hence, we obtain that the theory of a nilpotent three-form multiplet specified by eqs. (4.1a), (4.1b) arises as the infrared limit of the $\sigma$-model (4.3). Since $S_{\text {div }}=0$ on the solution (4.18) the action for the remaining fields $\psi, H, G$ comes from the subleading terms in the action (4.5)

$$
\begin{aligned}
S[\psi, H, G]=\int \mathrm{d}^{4} x[ & -\partial_{a}\left(\frac{\psi^{2}}{2 F}\right) \partial^{a}\left(\frac{\bar{\psi}}{2 \bar{F}}\right)+\frac{\mathrm{i}}{2} \partial_{\alpha \dot{\alpha}} \psi^{\alpha} \bar{\psi}^{\dot{\alpha}}-\frac{\mathrm{i}}{2} \psi^{\alpha} \partial_{\alpha \dot{\alpha}} \bar{\psi}^{\dot{\alpha}} \\
& +F \bar{F}-h(F+\bar{F})], \quad F=H+\mathrm{i} G .
\end{aligned}
$$

Note that this is just the component form of the superfield action (4.2). This action should be supplemented by the boundary term

$$
S_{\text {boundary }}[\psi, H, G]=-2 \int \mathrm{d}^{4} x \partial_{a}\left(G C^{a}\right) .
$$

The auxiliary fields $H$ and $G$ can be eliminated using their equations of motion which gives

$$
\begin{aligned}
& H-h-\frac{\bar{\psi}^{2}}{4 \bar{F}^{2}} \square \frac{\psi^{2}}{2 F}-\frac{\psi^{2}}{4 F^{2}} \square \frac{\bar{\psi}^{2}}{2 \bar{F}}=0, \\
& \partial_{a}\left[G+\mathrm{i} \frac{\bar{\psi}^{2}}{4 \bar{F}^{2}} \square \frac{\psi^{2}}{2 F}-\mathrm{i} \frac{\psi^{2}}{4 F^{2}} \square \frac{\bar{\psi}^{2}}{2 \bar{F}}\right]=0 .
\end{aligned}
$$

Eq. (4.21b) is solved by

$$
G+\frac{\mathrm{i}}{2}\left(\frac{\bar{\psi}^{2}}{2 \bar{F}^{2}} \square \frac{\psi^{2}}{2 F}-\frac{\psi^{2}}{2 F^{2}} \square \frac{\bar{\psi}^{2}}{2 \bar{F}}\right)=g
$$

for some constant $g$. Eqs. (4.21a) and (4.22) can also be written as

$$
F-\boldsymbol{f}-\frac{\bar{\psi}^{2}}{2 \bar{F}^{2}} \square \frac{\psi^{2}}{2 F}=0
$$

where, as before, $\boldsymbol{f}=h+\mathrm{i} g$.

\section{Models for spontaneously broken supergravity}

In describing supergravity-matter systems in superspace, it is useful, following the ideas pioneered in $[32,33]$, to formulate every pure supergravity theory as conformal supergravity coupled to a compensating supermultiplet. Different off-shell formulations for supergravity correspond to different compensators [21,34]. Conformal supergravity can be realised using the supergravity multiplet described in terms of the superspace geometry of [35, 36] (which underlies the Wess-Zumino approach [37] to the old minimal formulation for $\mathcal{N}=1$ supergravity developed independently in $[38,39]$ ) augmented with the super-Weyl invariance of [40]. An infinitesimal super-Weyl transformation has the form

$$
\begin{aligned}
\delta_{\sigma} \mathcal{D}_{\alpha} & =\left(\bar{\sigma}-\frac{1}{2} \sigma\right) \mathcal{D}_{\alpha}+\left(\mathcal{D}^{\beta} \sigma\right) M_{\alpha \beta} \\
\delta_{\sigma} \overline{\mathcal{D}}_{\dot{\alpha}} & =\left(\sigma-\frac{1}{2} \bar{\sigma}\right) \overline{\mathcal{D}}_{\dot{\alpha}}+\left(\overline{\mathcal{D}}^{\dot{\beta}} \bar{\sigma}\right) \bar{M}_{\dot{\alpha} \dot{\beta}} \\
\delta_{\sigma} \mathcal{D}_{\alpha \dot{\alpha}} & =\frac{1}{2}(\sigma+\bar{\sigma}) \mathcal{D}_{\alpha \dot{\alpha}}+\frac{\mathrm{i}}{2}\left(\overline{\mathcal{D}}_{\dot{\alpha}} \bar{\sigma}\right) \mathcal{D}_{\alpha}+\frac{\mathrm{i}}{2}\left(\mathcal{D}_{\alpha} \sigma\right) \overline{\mathcal{D}}_{\dot{\alpha}}+\left(\mathcal{D}^{\beta}{ }_{\alpha} \sigma\right) M_{\alpha \beta}+\left(\mathcal{D}_{\alpha} \dot{\beta} \bar{\sigma}\right) \bar{M}_{\dot{\alpha} \dot{\beta}},
\end{aligned}
$$

where $\sigma$ is an arbitrary covariantly chiral scalar superfield, $\overline{\mathcal{D}}_{\dot{\alpha}} \sigma=0$. 
There are several natural ways to lift the Goldstino superfield $V$, which was described in section 1, to supergravity. Of course, the constraints (1.1) are generalised to curved superspace in the unique way given in $[1,2]$ :

$$
\begin{aligned}
V^{2} & =0, \\
V \mathcal{D}_{A} \mathcal{D}_{B} V & =0, \\
V \mathcal{D}_{A} \mathcal{D}_{B} \mathcal{D}_{C} V & =0 .
\end{aligned}
$$

Non-uniqueness occurs when choosing a super-Weyl transformation for $V$. In this paper we consider the following transformation laws:

$$
\delta_{\sigma} V^{(\mathrm{I})}=0
$$

and

$$
\delta_{\sigma} V^{(\mathrm{II})}=(\sigma+\bar{\sigma}) V^{(\mathrm{II})} .
$$

In both cases, the constraints (5.2) are super-Weyl invariant. One can choose a more general super-Weyl transformation law for $V$ of the form $\delta_{\sigma} V=a(\sigma+\bar{\sigma}) V$, for some parameter $a$, by multiplying $V^{(\mathrm{I})}$ by some power of the compensator used. In particular, the choice $a=-1$ was made in [2] to describe the Goldstino brane.

\subsection{Super-Weyl inert $V$}

Let us assign the super-Weyl transformation law (5.3) to the Goldstino superfield $V$. Then the coupling of the old minimal supergravity [37-39] to $V$ is described by the super-Weyl invariant action [1]

$$
\begin{aligned}
S= & -\frac{3}{\kappa^{2}} \int \mathrm{d}^{4} x \mathrm{~d}^{2} \theta \mathrm{d}^{2} \bar{\theta} E \bar{S}_{0} S_{0}+\left\{\frac{\mu}{\kappa^{2}} \int \mathrm{d}^{4} x \mathrm{~d}^{2} \theta \mathcal{E} S_{0}^{3}+\text { c.c. }\right\} \\
& +\int \mathrm{d}^{4} x \mathrm{~d}^{2} \theta \mathrm{d}^{2} \bar{\theta} E\left\{\frac{1}{16} V \mathcal{D}^{\alpha}\left(\overline{\mathcal{D}}^{2}-4 R\right) \mathcal{D}_{\alpha} V-2 f \bar{S}_{0} S_{0} V\right\} .
\end{aligned}
$$

Here $S_{0}$ the chiral compensator, $\overline{\mathcal{D}}_{\dot{\alpha}} S_{0}=0$, with the super-Weyl transformation $\delta_{\sigma} S_{0}=$ $\sigma S_{0} \cdot{ }^{6}$ The functional in the first line of (5.5) is the old minimal supergravity action, in which the expression in the figure brackets is the supersymmetric cosmological term [42, 43].

Within the new minimal formulation for $\mathcal{N}=1$ supergravity [45] (see also [46, 47]), the compensator is a real covariantly linear scalar superfield,

$$
\left(\overline{\mathcal{D}}^{2}-4 R\right) \mathbb{L}=0, \quad \overline{\mathbb{L}}=\mathbb{L},
$$

with the super-Weyl transformation $\delta_{\sigma} \mathbb{L}=(\sigma+\bar{\sigma}) \mathbb{L}$. The action for supergravity coupled to the Goldstino superfield $V$ is [1]

$$
S=\int \mathrm{d}^{4} x \mathrm{~d}^{2} \theta \mathrm{d}^{2} \bar{\theta} E\left\{\frac{3}{\kappa^{2}} \mathbb{L} \ln \frac{\mathbb{L}}{\left|S_{0}\right|^{2}}+\frac{1}{16} V \mathcal{D}^{\alpha}\left(\overline{\mathcal{D}}^{2}-4 R\right) \mathcal{D}_{\alpha} V-2 f \mathbb{L} V\right\},
$$

\footnotetext{
${ }^{6}$ Here $E$ is the full superspace measure, and $\mathcal{E}$ denotes the chiral density. We use the notation $S_{0}$ for the chiral compensator following [34,41]. In the superspace literature, the chiral compensator is usually denoted $\Phi$, see e.g. section 6.6.1 in [24].
} 
where $S_{0}$ is a covariantly chiral scalar superfield, $\overline{\mathcal{D}}_{\dot{\alpha}} S_{0}=0$ (it is a purely gauge degree of freedom). The first term in (5.7) corresponds to the new minimal supergravity action. An important feature of unbroken new minimal supergravity is that it does not allow any supersymmetric cosmological term $[46,47] .^{7}$

\subsection{Super-Weyl non-invariant $V$}

If the Goldstino superfield $V$ is chosen to possess the super-Weyl transformation law (5.4), then we can associate with it the following three-form superfield

$$
\Psi=-\frac{1}{4}\left(\overline{\mathcal{D}}^{2}-4 R\right) V
$$

It is nilpotent, $\Psi^{2}=0$, as a consequence of (5.2). It transforms as a primary superfield under the super-Weyl group,

$$
\delta_{\sigma} \Psi=3 \sigma \Psi
$$

The action for old minimal supergravity coupled to the Goldstino superfield $\Psi$ is

$$
S=\int \mathrm{d}^{4} x \mathrm{~d}^{2} \theta \mathrm{d}^{2} \bar{\theta} E\left(-\frac{3}{\kappa^{2}} \bar{S}_{0} S_{0}+\frac{\bar{\Psi} \Psi}{\left(\bar{S}_{0} S_{0}\right)^{2}}\right)+\left\{\int \mathrm{d}^{4} x \mathrm{~d}^{2} \theta \mathcal{E}\left(\frac{\mu}{\kappa^{2}} S_{0}^{3}-f \Psi\right)+\text { c.c. }\right\} .
$$

This model is equivalent to (5.5).

\subsection{Three-form supergravity}

In 1981, Gates and Siegel [20] proposed a variant formulation of the old minimal supergravity, which is obtained from the supergravity action given in [48] by replacing the compensating chiral scalar superfield with a three-form superfield. ${ }^{8}$ At the component level, this supergravity formulation was elaborated in [50] where is was called "three-form supergravity." The modern prepotential formulation for three-form supergravity was given in appendix B of [51]. Further aspects of three-form supergravity were studied in [52].

Here we describe three-form supergravity following [51]. This supergravity formulation is obtained from the old minimal theory by replacing $S_{0}^{3}$ with

$$
\Xi^{3}=-\frac{1}{4}\left(\overline{\mathcal{D}}^{2}-4 R\right) P, \quad \bar{P}=P,
$$

where the prepotential $P$ is a real scalar superfield such that $\Xi$ is nowhere vanishing. The super-Weyl transformation of $P$ is defined to be

$$
\delta_{\sigma} P=(\sigma+\bar{\sigma}) P .
$$

\footnotetext{
${ }^{7}$ Among the known off-shell formulations for $\mathcal{N}=1$ supergravity (see [21, 24] for reviews), supersymmetric cosmological terms exist only for the old minimal supergravity [42, 43] (and its variant version reviewed in section 5.3 below) and the $n=-1$ non-minimal supergravity of [44].

${ }^{8}$ Probably the first serious study of matter three-form multiplets coupled to supergravity was given in [23]. A superform formulation for the three-form multiplet in a supergravity background was given in $[49]$.
} 
Then the chiral superfield $\Xi$ transforms as

$$
\delta_{\sigma} \Xi=\sigma \Xi
$$

The pure supergravity action is

$$
\begin{aligned}
S_{\mathrm{SG}} & =-\frac{3}{\kappa^{2}} \int \mathrm{d}^{4} x \mathrm{~d}^{2} \theta \mathrm{d}^{2} \bar{\theta} E \bar{\Xi} \Xi+\frac{2 m}{\kappa^{2}} \int \mathrm{d}^{4} x \mathrm{~d}^{2} \theta \mathrm{d}^{2} \bar{\theta} E P \\
& =-\frac{3}{\kappa^{2}} \int \mathrm{d}^{4} x \mathrm{~d}^{2} \theta \mathrm{d}^{2} \bar{\theta} E \bar{\Xi} \Xi+\frac{m}{\kappa^{2}}\left\{\int \mathrm{d}^{4} x \mathrm{~d}^{2} \theta \mathcal{E} \Xi^{3}+\text { c.c. }\right\} .
\end{aligned}
$$

Here the second term on the right is a supersymmetric cosmological term.

The supergravity action (5.14) is invariant under gauge transformations

$$
\delta P=L, \quad\left(\overline{\mathcal{D}}^{2}-4 R\right) L=0,
$$

which leave invariant the chiral superfield $\Xi^{3}$ defined by (5.11). The equation of motion for $P$ is

$$
\mathbb{R}+\overline{\mathbb{R}}=2 m, \quad \mathbb{R}:=-\frac{1}{4} \Xi^{-2}\left(\overline{\mathcal{D}}^{2}-4 R\right) \bar{\Xi} .
$$

Here the chiral scalar $\mathbb{R}$ is invariant under the super-Weyl transformations. The general solution to the equation of motion is $\mathbb{R}=m+\mathrm{i} e$, for some real parameter $e$.

\subsection{Three-form Goldstino superfield in supergravity}

The nilpotent three-form multiplet (4.1) is naturally lifted to supergravity. In curved superspace, its gauge-invariant field strength is defined by

$$
\mathcal{Y}=-\frac{1}{4}\left(\overline{\mathcal{D}}^{2}-4 R\right) \mathcal{U}, \quad \overline{\mathcal{U}}=\mathcal{U}
$$

This chiral scalar is constrained to be nilpotent,

$$
\mathcal{Y}^{2}=0
$$

in conjunction with the requirement that the descendant $\mathcal{D}^{2} \mathcal{Y}$ is nowhere vanishing. The prepotential $\mathcal{U}$ in $(5.17 \mathrm{a})$ is postulated to possess the super-Weyl transformation

$$
\delta_{\sigma} \mathcal{U}=(\sigma+\bar{\sigma}) \mathcal{U}
$$

which implies that $\mathcal{Y}$ is transforms as a super-Weyl primary superfield,

$$
\delta_{\sigma} \mathcal{Y}=3 \sigma \mathcal{Y} .
$$

The chiral scalar $\mathcal{Y}$ defined by (5.17a) is invariant under gauge transformations of $U$ of the type (5.15).

The action for old minimal supergravity coupled to the Goldstino superfield $\mathcal{Y}$ is

$$
S=\int \mathrm{d}^{4} x \mathrm{~d}^{2} \theta \mathrm{d}^{2} \bar{\theta} E\left(-\frac{3}{\kappa^{2}} \bar{S}_{0} S_{0}+\frac{\overline{\mathcal{Y}} \mathcal{Y}}{\left(\bar{S}_{0} S_{0}\right)^{2}}\right)+\left\{\int \mathrm{d}^{4} x \mathrm{~d}^{2} \theta \mathcal{E}\left(\frac{\mu}{\kappa^{2}} S_{0}^{3}-h \mathcal{Y}\right)+\text { c.c. }\right\} .
$$


In the flat superspace limit it reduces to (4.2). This action should be supplemented by a boundary term generalising (4.20).

As discussed in section 4, the Goldstino superfield $\mathcal{Y}$ contains two independent auxiliary fields, $F=H+\mathrm{i} G$, of which $H$ is a scalar and $G$ is the field strength of a gauge threeform. In supergravity, both $H$ and $G$ produce positive contributions to the cosmological constant. While the contribution from $H$ is uniquely determined by the parameter of the supersymmetry breaking $h$ in (5.20), the contribution from $G$ is dynamical. We believe that the latter may be used to neutralise the negative contribution from the supersymmetric cosmological term.

The idea that massless gauge three-forms make it possible to generate a cosmological constant dynamically, has attracted much interest since the early 1980s [30, 53-58]. We believe it will be useful in the framework of spontaneously broken local supersymmetry.

After this work had been submitted to the hep-th archive, we became aware of the recent paper [59] in which the nilpotent three-form multiplet was also studied and used for different purposes.

\section{Acknowledgments}

E.I.B. would like to thank the Physics Department at the University of Pennsylvania where some of this work was done for warm hospitality. The work of E.I.B. was supported in part by the ARC Future Fellowship FT120100466. Both authors were supported in part by the Australian Research Council, project No. DP140103925. The work of SMK was also supported in part by the Australian Research Council, project No. DP160103633.

Open Access. This article is distributed under the terms of the Creative Commons Attribution License (CC-BY 4.0), which permits any use, distribution and reproduction in any medium, provided the original author(s) and source are credited.

\section{References}

[1] S.M. Kuzenko, I.N. McArthur and G. Tartaglino-Mazzucchelli, Goldstino superfields in $\mathcal{N}=2$ supergravity, JHEP 05 (2017) 061 [arXiv: 1702.02423] [INSPIRE].

[2] I. Bandos, M. Heller, S.M. Kuzenko, L. Martucci and D. Sorokin, The Goldstino brane, the constrained superfields and matter in $\mathcal{N}=1$ supergravity, JHEP 11 (2016) 109 [arXiv: 1608.05908] [INSPIRE].

[3] U. Lindström and M. Roček, Constrained local superfields, Phys. Rev. D 19 (1979) 2300 [INSPIRE].

[4] M. Roček, Linearizing the Volkov-Akulov model, Phys. Rev. Lett. 41 (1978) 451 [INSPIRE].

[5] E.A. Ivanov and A.A. Kapustnikov, General relationship between linear and nonlinear realizations of supersymmetry, J. Phys. A 11 (1978) 2375 [INSPIRE].

[6] S.M. Kuzenko and S.J. Tyler, Complex linear superfield as a model for Goldstino, JHEP 04 (2011) 057 [arXiv: 1102.3042] [INSPIRE].

[7] P. Fayet and J. Iliopoulos, Spontaneously broken supergauge symmetries and Goldstone spinors, Phys. Lett. 51B (1974) 461 [INSPIRE]. 
[8] E. Dudas, S. Ferrara, A. Kehagias and A. Sagnotti, Properties of nilpotent supergravity, JHEP 09 (2015) 217 [arXiv: 1507.07842] [InSPIRE].

[9] E.A. Bergshoeff, D.Z. Freedman, R. Kallosh and A. Van Proeyen, Pure de Sitter supergravity, Phys. Rev. D 92 (2015) 085040 [arXiv:1507.08264] [INSPIRE].

[10] F. Hasegawa and Y. Yamada, Component action of nilpotent multiplet coupled to matter in 4 dimensional $\mathcal{N}=1$ supergravity, JHEP 10 (2015) 106 [arXiv:1507.08619] [INSPIRE].

[11] S.M. Kuzenko, Complex linear Goldstino superfield and supergravity, JHEP 10 (2015) 006 [arXiv:1508.03190] [INSPIRE].

[12] P.K. Townsend, Cosmological constant in supergravity, Phys. Rev. D 15 (1977) 2802 [INSPIRE].

[13] I. Bandos, L. Martucci, D. Sorokin and M. Tonin, Brane induced supersymmetry breaking and de Sitter supergravity, JHEP 02 (2016) 080 [arXiv:1511.03024] [INSPIRE].

[14] D.V. Volkov and V.A. Soroka, Higgs effect for Goldstone particles with spin 1/2, JETP Lett. 18 (1973) 312 [Pisma Zh. Eksp. Teor. Fiz. 18 (1973) 529] [INSPIRE].

[15] D.V. Volkov and V.A. Soroka, Gauge fields for symmetry group with spinor parameters, Theor. Math. Phys. 20 (1974) 829 [inSPIRE].

[16] S. Deser and B. Zumino, Broken supersymmetry and supergravity, Phys. Rev. Lett. 38 (1977) 1433 [INSPIRE].

[17] R. Casalbuoni, S. De Curtis, D. Dominici, F. Feruglio and R. Gatto, Nonlinear realization of supersymmetry algebra from supersymmetric constraint, Phys. Lett. B 220 (1989) 569 [INSPIRE].

[18] Z. Komargodski and N. Seiberg, From linear SUSY to constrained superfields, JHEP 09 (2009) 066 [arXiv: 0907.2441] [INSPIRE].

[19] S.J. Gates, Jr., Super p form gauge superfields, Nucl. Phys. B 184 (1981) 381 [INSPIRE].

[20] S.J. Gates, Jr. and W. Siegel, Variant superfield representations, Nucl. Phys. B 187 (1981) 389 [INSPIRE].

[21] S.J. Gates, M.T. Grisaru, M. Roček and W. Siegel, Superspace or one thousand and one lessons in supersymmetry, Front. Phys. 58 (1983) 1 [hep-th/0108200] [INSPIRE].

[22] I.A. Batalin and G.A. Vilkovisky, Quantization of gauge theories with linearly dependent generators, Phys. Rev. D 28 (1983) 2567 [Erratum ibid. D 30 (1984) 508] [INSPIRE].

[23] I.L. Buchbinder and S.M. Kuzenko, Quantization of the classically equivalent theories in the superspace of simple supergravity and quantum equivalence, Nucl. Phys. B 308 (1988) 162 [INSPIRE].

[24] I.L. Buchbinder and S.M. Kuzenko, Ideas and methods of supersymmetry and supergravity or a walk through superspace, IOP, Bristol, U.K. (1995), revised edition (1998).

[25] D.V. Volkov and V.P. Akulov, Possible universal neutrino interaction, JETP Lett. 16 (1972) 438 [Pisma Zh. Eksp. Teor. Fiz. 16 (1972) 621] [inSPIRE].

[26] D.V. Volkov and V.P. Akulov, Is the neutrino a Goldstone particle?, Phys. Lett. 46B (1973) 109 [INSPIRE].

[27] V.P. Akulov and D.V. Volkov, Goldstone fields with spin 1/2, Theor. Math. Phys. 18 (1974) 28 [Teor. Mat. Fiz. 18 (1974) 39] [InSPIRE].

[28] S.M. Kuzenko and S.J. Tyler, On the Goldstino actions and their symmetries, JHEP 05 (2011) 055 [arXiv:1102.3043] [INSPIRE]. 
[29] S.M. Kuzenko and I.N. McArthur, Goldstino superfields for spontaneously broken $N=2$ supersymmetry, JHEP 06 (2011) 133 [arXiv:1105.3001] [INSPIRE].

[30] M.J. Duncan and L.G. Jensen, Four forms and the vanishing of the cosmological constant, Nucl. Phys. B 336 (1990) 100 [inSPIRE].

[31] K. Groh, J. Louis and J. Sommerfeld, Duality and couplings of 3-form-multiplets in $N=1$ supersymmetry, JHEP 05 (2013) 001 [arXiv:1212.4639] [INSPIRE].

[32] W. Siegel, A polynomial action for a massive, self-interacting chiral superfield coupled to supergravity, HUTP-77/A077 (1977).

[33] M. Kaku and P.K. Townsend, Poincaré supergravity as broken superconformal gravity, Phys. Lett. 76B (1978) 54 [inSPIRE].

[34] S. Ferrara, L. Girardello, T. Kugo and A. Van Proeyen, Relation between different auxiliary field formulations of $N=1$ supergravity coupled to Matter, Nucl. Phys. B 223 (1983) 191 [INSPIRE].

[35] R. Grimm, J. Wess and B. Zumino, Consistency checks on the superspace formulation of supergravity, Phys. Lett. 73B (1978) 415 [INSPIRE].

[36] R. Grimm, J. Wess and B. Zumino, A complete solution of the Bianchi identities in superspace, Nucl. Phys. B 152 (1979) 255 [INSPIRE].

[37] J. Wess and B. Zumino, Superfield lagrangian for supergravity, Phys. Lett. 74B (1978) 51 [INSPIRE].

[38] K.S. Stelle and P.C. West, Minimal auxiliary fields for supergravity, Phys. Lett. 74B (1978) 330 [INSPIRE].

[39] S. Ferrara and P. van Nieuwenhuizen, The auxiliary fields of supergravity, Phys. Lett. 74B (1978) 333 [INSPIRE].

[40] P.S. Howe and R.W. Tucker, Scale invariance in superspace, Phys. Lett. 80B (1978) 138 [INSPIRE].

[41] T. Kugo and S. Uehara, Improved superconformal gauge conditions in the $N=1$ supergravity Yang-Mills matter system, Nucl. Phys. B 222 (1983) 125 [INSPIRE].

[42] S. Ferrara and P. van Nieuwenhuizen, Tensor calculus for supergravity, Phys. Lett. B 76 (1978) 404.

[43] W. Siegel, Solution to constraints in Wess-Zumino supergravity formalism, Nucl. Phys. B 142 (1978) 301 [INSPIRE].

[44] D. Butter and S.M. Kuzenko, A dual formulation of supergravity-matter theories, Nucl. Phys. B 854 (2012) 1 [arXiv: 1106.3038] [INSPIRE].

[45] M.F. Sohnius and P.C. West, An alternative minimal off-shell version of $N=1$ supergravity, Phys. Lett. 105B (1981) 353 [INSPIRE].

[46] M.F. Sohnius and P.C. West, The new minimal formulation of $N=1$ supergravity and its tensor calculus, in Quantum structure of space and Time, M.J. Duff and C.J. Isham eds., Cambridge University Press, Cambridge U.K. (1982).

[47] M. Sohnius and P.C. West, The tensor calculus and matter coupling of the alternative minimal auxiliary field formulation of $N=1$ supergravity, Nucl. Phys. B 198 (1982) 493 [INSPIRE].

[48] W. Siegel and S.J. Gates Jr., Superfield supergravity, Nucl. Phys. B 147 (1979) 77 [inSPIRE]. 
[49] P. Binetruy, F. Pillon, G. Girardi and R. Grimm, The three form multiplet in supergravity, Nucl. Phys. B 477 (1996) 175 [hep-th/9603181] [InSPIRE].

[50] B.A. Ovrut and D. Waldram, Membranes and three form supergravity, Nucl. Phys. B 506 (1997) 236 [hep-th/9704045] [INSPIRE].

[51] S.M. Kuzenko and S.A. McCarthy, On the component structure of $N=1$ supersymmetric nonlinear electrodynamics, JHEP 05 (2005) 012 [hep-th/0501172] [INSPIRE].

[52] I.A. Bandos and C. Meliveo, Supermembrane interaction with dynamical $D=4 N=1$ supergravity. Superfield Lagrangian description and spacetime equations of motion, JHEP 08 (2012) 140 [arXiv:1205.5885] [inSPIRE].

[53] V. Ogievetsky and E. Sokatchev, Equation of motion for the axial gravitational superfield, Sov. J. Nucl. Phys. 32 (1980) 589 [Yad. Fiz. 32 (1980) 1142] [inSPIRE].

[54] M.J. Duff and P. van Nieuwenhuizen, Quantum inequivalence of different field representations, Phys. Lett. B 94 (1980) 179.

[55] A. Aurilia, H. Nicolai and P.K. Townsend, Hidden constants: the theta parameter of $Q C D$ and the cosmological constant of $N=8$ supergravity, Nucl. Phys. B 176 (1980) 509 [INSPIRE].

[56] S.W. Hawking, The cosmological constant is probably zero, Phys. Lett. B 134 (1984) 403.

[57] M.J. Duff, The cosmological constant is possibly zero, but the proof is probably wrong, Phys. Lett. B 226 (1989) 36 [INSPIRE].

[58] R. Bousso and J. Polchinski, Quantization of four form fluxes and dynamical neutralization of the cosmological constant, JHEP 06 (2000) 006 [hep-th/0004134] [INSPIRE].

[59] F. Farakos, A. Kehagias, D. Racco and A. Riotto, Scanning of the supersymmetry breaking scale and the gravitino mass in supergravity, JHEP 06 (2016) 120 [arXiv:1605.07631] [INSPIRE]. 